\title{
Expertise in Professional Software Design: A Process Study
}

\author{
Sabine Sonnentag \\ University of Amsterdam
}

\begin{abstract}
Forty professional software designers participated in a study in which they worked on a software design task and reported strategies for accomplishing that task. High performers were identified by a peer-nomination method and performance on a design. Verbal protocol analysis based on a comparison of 12 high and 12 moderate performers indicated that high performers structured their design process by local planning and showed more feedback processing, whereas moderate performers were more engaged in analyzing requirements and verbalizing task-irrelevant cognitions. High performers more often described problem comprehension and cooperation with colleagues as useful strategies. High and moderate performers did not differ with respect to length of experience. None of the differences between the two performance groups could be explained by length of experience.
\end{abstract}

Starting with de Groot's (1978) early study on worldclass chess players, expertise research became a quickly expanding research area within cognitive psychology (Chi, Glaser, \& Farr, 1988; Ericsson \& Smith, 1991b). More recently, Ford and Kraiger (1995) stressed the importance of expertise research for applied purposes. When designing training and personnel selection procedures, organizations need detailed information about skills and other characteristics required in the work process. In this context, identifying characteristics of expert professionals is extremely useful.

Ericsson and Smith (1991a) characterized the goal of expertise research as being able to "understand and account for what distinguishes outstanding individuals in a domain from less outstanding individuals" (p. 2). Thus, on the conceptual level expertise is equated with high and exceptional performance. However, within most studies, advanced students or professionals within a domain were

This research was supported by a grant from the German Research Community (DFG; So 295/1-1 and 1-2) that is gratefully acknowledged. I thank Dieter Ronge, Arndt Sommer, and Rabea Munzert for their help in categorizing the data and Michael Frese and Doris Fay for helpful comments on an earlier version of this report. Preliminary results of this study were presented at the Annual Meeting of the Academy of Management in Cincinnati, Ohio, August 1996, and at the 26th International Congress of Psychology in Montreal, Quebec, Canada, August 1996.

Correspondence concerning this article should be addressed to Sabine Sonnentag, Department of Psychology, University of Amsterdam, Roetersstraat 15, NL-1018 WB Amsterdam, the Netherlands. Electronic mail may be sent to ao_sonnentag@ macmail.psy.uva.nl. regarded as the experts whose cognitive processes and knowledge structures were compared with those of beginner students and other less experienced persons, the socalled novices (e.g., Chi, Glaser, \& Rees, 1982; Jeffries, Turner, Polson, \& Atwood, 1981; Patel \& Groen, 1991). Such comparisons fall short in explaining high and exceptional performance among professionals. In many domains, substantial performance differences exist among professionals not varying in length of experience (Reif \& Allen, 1990; Simmons \& Lunetta, 1993; Sonnentag, 1995).

This article describes a study on expertise in professional software design. To expand psychological knowledge about expertise and to be relevant for applied settings, expertise was operationalized as high performance and not as length of experience. Whether a long period of experience corresponds to high performance was a question to be answered on the basis of empirical data. In addition to the analysis of the overall amount of time spent for specific activities, the study aimed at gaining insight into action processes.

\section{Characteristics of High Performers}

Action theory offers a useful framework for analyzing actions and explaining high performance (Frese \& Zapf, 1994; Hacker, 1986). Following the action theory perspective, adequate problem comprehension, planning, feedback processing, task focus, use of visualizations, and knowledge of strategies can be regarded as essential characteristics of high performers and their action processes.

\section{Problem Comprehension}

Problem comprehension aims at the development of an adequate problem representation and is therefore an 
essential part of the problem-solving process. By gathering information about the task and relevant situational aspects, problem comprehension activities form the basis for planning and executing necessary action steps. Therefore, extensive problem comprehension activities and adequate problem representation are regarded as essential for high performance (Glaser \& Chi, 1988; Green \& Gilhooly, 1992). Empirical research in various domains showed that high performers, compared with others, spent significantly more time on problem comprehension and related activities (Klemp \&.McClelland, 1986; Lesgold et al., 1988; Schaub \& Strohschneider, 1992; Vessey, 1986). However, some studies reported contrary findings with more problem comprehension activities being typical for a lower performance level (Dörner, Kreuzig, Reither, \& Stäudel, 1983; Hershey, Walsh, Read, \& Chulef, 1990). A closer examination of these studies suggests that moderate performers increased their problem comprehension activities late in the problem solving process, which can be explained by an inability to build an adequate problem representation early in the process (Green \& Gilhooly, 1992; Klein \& Hoffman, 1993). Taken together, research indicates that early problem comprehension is crucial for successful problem solving. It can be assumed that high performing software designers are more engaged in problem comprehension activities than are moderate performers, particularly in the beginning of task accomplishment.

Hypothesis 1: High performers will spend more time on problem comprehension early in the process than will moderate performers.

Existing research has concentrated mainly on the amount of problem comprehension (Glaser \& Chi, 1988; Green \& Gilhooly, 1992). However, within applied research relatively little is known about the problem comprehension process. Therefore, the present study aimed additionally at a deeper analysis of the problem comprehension process. One aim was to explore whether the way persons arrive at an understanding of a problem differs across performance levels.

\section{Planning}

During planning, decisions about future action steps and their sequence are made (Hacker, 1986; Hayes-Roth \& Hayes-Roth, 1979; Miller, Galanter, \& Pribram, 1960). Planning is an important prerequisite for successful task accomplishment because by planning, difficulties and possible solutions can be anticipated. For example, how to deal with situational constraints, such as time limits or scarce resources, can be decided. Furthermore, planning implies forming intentions that help in initiating goalrelevant actions (cf. Gollwitzer, 1993). Previous research with student subjects as well as managers and service workers showed a positive relationship between amount of planning and performance (Dörner et al., 1983; Earley, Wojnaroski, \& Prest, 1987; Klemp \& McClelland, 1986). Therefore, it is assumed that high-performing software designers spend more time on planning than do moderate performers.

Hypothesis 2: High performers will spend more time on planning than will moderate performers.

\section{Feedback Processing}

Feedback processing comprises a comparison between a present situation and the cognitive representation of the goal. Processing the feedback allows one to evaluate how far a pursued goal has been achieved. In case of a discrepancy between a goal and the present situation, new action steps can be started (Carver \& Scheier, 1982). Thus, feedback processing is helpful in accomplishing the task. Research shows that actively seeking feedback is positively related to good performance in problem-solving tasks. For example, Dörner et al. (1983) found that high performers working on a complex task did not develop more hypotheses but evaluated the correctness of their hypotheses more often. This finding is consistent with results of a study by Simmons and Lunetta (1993) who reported that highperforming biologists more often processed feedback by questioning the correctness of their actions; lower performing biologists did not justify their actions. Similarly, Rohwer and Thomas (1989) found more continuous performance monitoring in highly performing participants. Given the feedback processing activity of high performers during software design, one can expect that high-performing software designers would spend more time evaluating their design solutions than do moderate performers.

Hypothesis 3: High performers will spend more time for feedback processing than moderate performers.

\section{Task Focus}

The hypotheses concerning problem comprehension, planning, and feedback processing refer to specific ontask activities. However, it can not be taken for granted that high and moderate performers differ only with respect to the amount of time spent on these specific on-task activities. An additional difference between the two groups might be seen in the extent in which they are engaged in on-task versus off-task activities. Ruminative and task-irrelevant cognitions can be regarded as typical examples of off-task activities in the context of problem solving and design tasks. Research on rumination shows that task-irrelevant thoughts are positively related to failure and low performance (Mikulincer, 1994; Mikulincer, Kedem, \& Zilcha-Segal, 1989). Therefore, with respect to software design, it is assumed that high performers spend less time on task-irrelevant cognitions than do moderate performers. 
Hypothesis 4: High performers will spend less time on task-irrelevant cognitions than will moderate performers.

Previous expertise research paid little attention to taskirrelevant cognitions (for an exception, see Shanteau, 1988). As very little is known about how task-irrelevant cognitions are handled during the working process, the current study examined whether high and moderate performers differ with respect to the way they deal with taskirrelevant cognitions.

\section{Visualizations}

Empirical studies show that visualizations (i.e., graphical representations such as sketches and diagrams) play an important role in design and related activities (Bellamy, 1994; Black, 1990). Visualizations can directly support the problem-solving process by displaying all information needed in a condensed way (Larkin \& Simon, 1987). Although researchers agree about the importance of visualizations, empirical research has not yet answered the question unequivocally on whether high or moderate performers rely more on visualizations (Adelson \& Soloway, 1988; Chi et al., 1982; Davies, 1992). Two arguments speak for a higher visualization activity of high performers: First, visualizations help in arriving at an adequate problem representation (Anzai, 1991; Chi et al., 1982). Second, because visualizations show graphically possible flaws or inconsistencies inherent in design solutions, they can serve as an important basis for later feedback processing. Assuming that high performers aim at optimizing problem representation and feedback processing (Glaser \& Chi, 1988; Green \& Gilhooly, 1992; Simmons \& Lunetta, 1993), it is likely that they will produce more visualizations during problem comprehension and solution development than will moderate performers.

Hypothesis 5: High performers will produce more visualizations than moderate performers.

\section{Knowledge of Strategies}

Studies on knowledge differences between experts and nonexperts belong to the core of expertise research. Most studies compared experienced with less experienced persons (Chi et al., 1982; Soloway, Adelson, \& Ehrlich, 1988; Voss, Greene, Post, \& Penner, 1983). Thus, most of this research focused on knowledge effects of experience rather than of high performance. Furthermore, declarative knowledge was studied more often than procedural knowledge (Chase \& Simon, 1973; McKeithen, Reitman, Rueter, \& Hirtle, 1981). The question arises whether knowledge differences hold for high versus moderate performers and whether such differences exist also for procedural knowledge. Research on complex problem solving suggests that high and moderate performers use different strategies for task accomplishment (Dörner \& Schölkopf, 1991). Therefore, one can assume that high performers have a more comprehensive knowledge of successful strategies (Frese \& Zapf, 1994; Hacker, 1986, 1992). Models within industrial and organizational psychology specifying procedural knowledge as an important determinant of performance support this contention (Campbell, McCloy, Oppler, \& Sager, 1993).

Hypothesis 6: Compared with moderate performers, high performers will know more about strategies.

\section{Length of Experience}

Previous expertise research has shown that length of experience is an important variable when comparing students with professionals (Adelson, 1981; Eteläpelto, 1993; Jeffries et al., 1981). However, only a few expertise studies examined whether length of experience matters within samples of professionals. Some of these studies found that experienced professionals differed from less experienced colleagues with respect to performance and working processes (Koubek \& Salvendy, 1991; Wiggins \& O'Hare, 1995), whereas other studies did not (Charness, Krampe, \& Mayr, 1996; Ericsson, Krampe, \& Tesch-Römer, 1993; Sonnentag, 1995). Thus, the question is not yet answered whether length of experience plays as crucial a role for performance differences within professionals as it does for differences between students and professionals. Therefore, the present study examined whether length of experience can explain differences in performance level.

\section{Method}

\section{Participants}

Forty professional software designers participated in the study. They were recruited from 16 professional software development teams in Germany. These teams produced information and communication systems as well as software for logistic and process control purposes. One team was engaged in developing an expert system. Participants had a mean professional experience of 6.9 years ( $S D=2.9$ ) ranging from 3 to 14 years. During their careers, participants had worked with an average of 3.1 different programming languages ( $S D=1.6$ ). Mean age was 33.3 years $(S D=5.1)$. Thirteen $(32.5 \%)$ of the participants were female. Software designers were paid for their participation in the study. Payment was not contingent on task performance. ${ }^{1}$

For analyzing differences between high and moderate performers, 24 out of 40 participants were selected on the basis of peer nominations and design task performance (see below).

\footnotetext{
${ }^{1}$ The sample of this study was partially overlapping with the sample studied by Sonnentag (1996).
} 


\section{Material}

As a software design task, the lift control problem was chosen. The goal of this task is to design a software system that controls the movement of $\mathrm{N}$ lifts between $\mathrm{M}$ floors whereby a number of constraints must be taken into account (see the Appendix for a full description and English translation of the task). The lift control problem can be characterized as an ill-structured and knowledge-rich task specified by an informal, incomplete, and ambiguous description (Guindon, 1990). It therefore incorporates the essential features of a realistic software design task.

\section{Procedure}

The first part of the study consisted of a peer-nomination procedure conducted in the 16 participating software development teams. In the second part of the study, software designers participated in individual sessions in which they worked on the lift control problem. Participants had 120 min to complete this task. They were told that they did not need to write a detailed program but should produce a design solution that they could hand to an experienced programmer who would implement the program. The instruction stressed that the main "thinking work" should be incorporated in the participants' design solutions. No restrictions with respect to design method, programming language, and notations were made. Participants were provided with pens of different colors and as much paper as they needed. They were allowed to write down everything they wished, including sketches, notes, and questions to themselves. Participants were asked to think aloud while working on the task. The following verbalization instruction was used: "I am not only interested in the solution you arrive at, but also how you arrive there. Therefore, I would like you to think aloud while working on this task. What does thinking aloud mean? Take it literally. Think/reflect (Überlegen Sie) with a loud voice. Please verbalize everything that is in your mind while you are busy with the task, even if it might seem unimportant, including thoughts and ideas that at first glance might have nothing to do with the task. It is important that you do not judge your thoughts before verbalizing them."' When participants stopped verbalizing for more than $15 \mathrm{~s}$ they were prompted to continue. Verbalizations were tape-recorded and later transcribed fully. After having completed the design task, participants were interviewed about the strategies they followed and about their knowledge of strategies.

The task description, all instructions, verbalizations, and reported strategies were in German.

\section{Identification of High and Moderate Performers}

In a peer-nomination procedure every member of the 16 participating software teams was asked individually to indicate whom in the team he or she regarded as a "very good software professional." Subsequently, it was computed how often each software designer in the participating teams was nominated by his or her peers. To make sure that there was a certain amount of agreement among team members about the team's very good software professional, only software designers who were named by at least two of their peers were considered as possibly high performers. Software designers who were not named at all were considered as possibly moderate performers.

The validity of the peer nomination measure was tested by analyzing participants' design task performance. Participants' hand-written design solutions produced for the lift control problem were type-written and given a standard format. Raters blind to peer nomination outcomes and to the course of participants' design processes evaluated these type-written, standard format design solutions on the basis of four design criteria (algorithm quality, modularity, comprehensibility, and detail) on 5-point Likert scales. The four design criteria were combined into one measure of design task performance (Cronbach's $\alpha=.79$ ). Twenty designs were evaluated by two independent raters. Interrater agreement for the combined measure of design task performance was $r=.87$. Analysis showed that participants nominated by at least two peers showed a better design-task performance $(M=3.1, S D=0.8)$ than participants not nominated at all $(M=2.4, S D=0.9 ; t(38)=2.54, p<.05 ; d=$ $0.80)$, indicating convergent validity of peer nomination and design task performance.

To arrive at a performance measure with high external and internal validity, peer nomination outcome and design task performance were combined into one measure: Twelve participants who were named at least twice in the peer nomination procedure and who showed a design task performance above the median were regarded as high performers. ${ }^{2}$ Twelve participants who were not named at all in the peer-nomination procedure and who showed a design task performance below the median were regarded as moderate performers.

\section{Dependent Measures}

With respect to the design task, three types of dependent measures were assessed: verbal protocol data, visualization data, and knowledge about strategies. Additionally, length of experience was measured. Means, standard deviations, and intercorrelations of all dependent measures are shown in Table 1. All raters involved in the coding process were unaware of peer-nomination outcomes and participants' design task performance.

Verbal protocol data. Tape-recorded thinking-aloud protocols were verbally transcribed and segmented. A complete phrase constituted one segment. In the case of incomplete phrases, the incomplete parts of a phrase were regarded as the segment unit. The average length of a transcribed verbal protocol was 491 segments $(S D=185)$.

Verbalizations were categorized segment by segment using a category system comprising problem comprehension, planning, feedback processing, task-irrelevant cognitions, comments, and solution development. Problem comprehension was operationalized by two categories: problem comprehension by analyzing

\footnotetext{
${ }^{2}$ Originally, 15 participants named in the peer-nomination procedure as a "very good software professional" showed a design task above the median. To keep the high and moderate performance groups equal in size, I regarded only the 12 peernominated participants with the highest design task performance as high performers in further analyses.
} 
Table 1

Means, Standard Deviations, and Intercorrelations of Dependent Measures

\begin{tabular}{|c|c|c|c|c|c|c|c|c|c|c|c|c|c|}
\hline Measure & $M$ & $S D$ & 1 & 2 & 3 & 4 & 5 & 6 & 7 & 8 & 9 & 10 & 11 \\
\hline \multicolumn{14}{|l|}{$\%$ of verbal protocols } \\
\hline 1. PC by requirements & 15.9 & 8.7 & $(.87)$ & & & & & & & & & & \\
\hline 2. PC by scenarios & 5.6 & 4.7 & .05 & $(.96)$ & & & & & & & & & \\
\hline 3. Planning ahead & 1.6 & 1.4 & .01 & -.18 & $(.69)$ & & & & & & & & \\
\hline 4. Local planning & 4.3 & 2.1 & .03 & -.07 & $.38^{*}$ & $(.82)$ & & & & & & & \\
\hline 5. Feedback processing & 10.9 & 8.2 & -.27 & -.24 & -.18 & .02 & $(.68)$ & & & & & & \\
\hline 6. Task-irrelevant cognitions & 2.8 & 4.1 & .01 & -.12 & .28 & -.19 & $-.41^{* *}$ & $(.81)$ & & & & & \\
\hline 7. Comments & 5.8 & 4.2 & -.13 & .03 & $.41 * *$ & .27 & -.27 & $.43 * *$ & $(.72)$ & & & & \\
\hline 8. Solution development & 52.0 & 10.0 & $-.56 * *$ & -.30 & -.26 & -.30 & -.14 & -.16 & $-.35^{*}$ & (.86) & & & \\
\hline \multicolumn{14}{|l|}{ Visualizations } \\
\hline 9. Problem visualizations & 0.75 & 0.44 & -.02 & $.32 *$ & -.01 & $-.37^{*}$ & -.11 & .04 & -.14 & .04 & & & \\
\hline 10. Solution visualizations & 0.30 & 0.46 & -.14 & .21 & .00 & .22 & .27 & -.25 & .06 & -.15 & .00 & & \\
\hline \multicolumn{14}{|l|}{ Knowledge } \\
\hline 11. No. of strategies & 3.0 & 1.9 & $-.32 *$ & .13 & .08 & .19 & $.41^{*}$ & $-.35 *$ & -.14 & .04 & .07 & $.38^{*}$ & \\
\hline \multicolumn{14}{|l|}{ Experience } \\
\hline 12. Length of experience & 6.9 & 2.9 & .01 & .24 & -.14 & -.12 & -.04 & -.09 & -.10 & .02 & .09 & .00 & .00 \\
\hline
\end{tabular}

Note. $N=40$. PC by requirements = problem comprehension by analyzing requirements; PC by scenarios $=$ problem comprehension by scenarios. Interrater agreements are in parentheses.

$* p<.05 . * *<.01$

requirements (i.e., reading the requirements given in the task description and reflecting on them) and problem comprehension by scenarios (i.e., reflecting on typical problems and scenarios within the lift domain). Similarly, two planning categories were differentiated: planning ahead (i.e., reflecting and deciding on the future course of action; thinking about what to do first and what to postpone), and local planning (i.e., thinking about the next step without extensively reflecting on it). There was one category for feedback processing (i.e., evaluating the designed solution) and one for task-irrelevant cognitions (i.e., statements having nothing to do with the lift control task). Although no hypotheses had been formulated with respect to the categories comments (i.e., commenting on their own working process ) and solution development (i.e., designing the outline of the software system), they were included to ensure a complete categorization of the verbal protocols.

To examine the design process at a more detailed level and to test Hypothesis 1 which predicted problem comprehension differences only early in the process, I divided the total working time of each participant into four equal time intervals, resulting in four phases with a length of $30 \mathrm{~min}$ each. For these four phases, the percentage of each verbal protocol category was also computed.

For reliability analysis, 20 verbal protocols were categorized by two independent raters both at the segment and the protocol level. At the segment level, the first rater's categorization of every statement was compared with the second rater's categorization of that statement. Following this procedure, the average agreement of the two raters was $77.5 \%$. The corresponding Cohen's kappa of .65 can be regarded as satisfactory (Landis \& Koch, 1977). Reliability analysis at the protocol level was based on the respective percentages for every category provided by the first and second rater. Correlations ranged from $r=.68$ (feedback processing) to $r=.96$ (problem comprehension by scenarios) with a median correlation of $r=.82$ for all seven categories. All further analyses were based on the categorizations provided by the more experienced rater.

Visualization data. Visualization data were based on graphical representations and sketches produced by the participants. A differentiation was made between problem visualizations and solution visualizations. Sketches showing houses, lifts, and their buttons were coded as problem visualizations. Graphical representations of data structures or relationships between separate modules were coded as solution visualizations. Both visualization measures were dichotomous. Additionally, the total amount of notes taken was assessed by computing the number of pages participants had filled with notes, visualizations, and descriptions of the design solution. This variable was used later as a control variable.

Knowledge about strategies. For assessing knowledge about strategies, a procedure suggested by Wolff (1989) was adopted: Participants were asked to imagine an inexperienced colleague having to work on the lift control problem and to describe strategies they would recommend to this inexperienced colleague. Strategies were referred to as "higher order principles" and illustrated by an example: "A higher order principle could be to start with the most simple part of the task-or to start with the most difficult part." Strategies recommended by the participants were verbally recorded. The number of recommended strategies was computed and used as knowledge measure in the analysis.

One might argue that the number of recommended strategies does not only reflect knowledge but also verbalization skills. To rule out this interpretation, an index for verbalization skills was computed and used subsequently as a covariate. Participants were asked which strategies (again referred to as higher order principles) they had used while working on the lift control problem and which strategies (higher order principles) they usually pursued in their everyday work situations. The verbalization skills index was computed by averaging the number of 
these two types of reported strategies $(r=31, N=40, p=$ $.054)$.

In addition to the number of recommended strategies, the content of these strategies was analyzed using a fine-grained category system developed by Sonnentag (1996). Strategies recommended by 20 participants were categorized by two raters resulting in an interrater agreement of $85.3 \%$ (Cohen's $\kappa=$ $.84)$. For comparing high and moderate performers the following strategies named by at least $25 \%$ of the total sample $(N=$ 40 ) were analyzed: intensive problem comprehension, cooperation with colleagues, and "divide and conquer" (i.e., divide the overall problem into subproblems and work on each of these sub-problems subsequently).

Length of experience. Participants indicated the length of their professional experience.

\section{Results}

\section{Test of Hypotheses}

Table 2 presents means and standard deviations of the verbal protocol categories for the two performance groups, both separately and averaged over the four phases. For every verbal protocol category a $2 \times 4$ analysis of variance (ANOVA) was used with performance level as a between-subjects variable and phase as a repeated variable (within-subject). ANOVA results are provided in Table 3 .

With respect to "problem comprehension by analyzing requirements," the ANOVA across all four phases showed that high performers spent less time analyzing requirements than did moderate performers. In both groups, ana- lyzing requirements declined over time with the highest number of requirement verbalizations in the first phase. For testing Hypothesis 1, "problem comprehension by analyzing requirements" in Phase 1 and Phase 2 was analyzed by performing a $2 \times 2$ ANOVA with phase as a repeated measurement variable. No differences between high and moderate performers were found, $F(1,22)=$ 2.13, $n s, d=-0.60$. Analysis of "problem comprehension by requirements" in Phase 3 and Phase 4 indicated that moderate performers spent more time analyzing requirements in these last two phases, $F(1,22)=5.40, p$ $<.05, d=-0.95$. This finding suggests that moderate performers' higher amount of overall "problem comprehension by analyzing requirements" is mainly due to their extensive requirement analysis late in the process. Over the four phases, high and moderate performers did not differ in "problem comprehension by scenarios." Separate ANOVAs for the first two phases, $F(1,22)=0.50$, $n s, d=0.29$, and the last two phases, $F(1,22)=0.96$, $n s, d=0.39$, did not result in significant differences between the two performance groups. Thus, no support for Hypothesis 1 was found.

Hypothesis 2 predicted that compared with moderate performers, high performers spend more time on planning. However, ANOVA showed no difference between these two groups with respect to planning ahead. For local planning a significant main effect of the repeated-measurement variable and a significant interaction effect were found. Thus, the amount of local planning differed in the course of the design process, with high and moderate

Table 2

Means and Standard Deviations of Verbal Protocol Categories in Four Phases

\begin{tabular}{|c|c|c|c|c|c|c|c|c|c|c|}
\hline \multirow[b]{2}{*}{ Measure } & \multicolumn{2}{|c|}{ Phase 1} & \multicolumn{2}{|c|}{ Phase 2} & \multicolumn{2}{|c|}{ Phase 3} & \multicolumn{2}{|c|}{ Phase 4} & \multicolumn{2}{|c|}{$\begin{array}{l}\text { All four } \\
\text { phases }\end{array}$} \\
\hline & $M$ & $S D$ & $M$ & $S D$ & $M$ & $S D$ & $M$ & $S D$ & $M$ & $S D$ \\
\hline \multicolumn{11}{|l|}{ High performers $(n=12)$} \\
\hline PC by requirements & 39.9 & 14.8 & 9.3 & 8.7 & 2.4 & 2.4 & 1.9 & 2.0 & 13.3 & 5.2 \\
\hline PC by scenarios & 11.5 & 12.5 & 8.2 & 9.9 & 6.2 & 5.5 & 4.3 & 5.8 & 7.4 & 6.5 \\
\hline Planning ahead & 2.3 & 2.9 & 2.6 & 3.7 & 1.9 & 2.1 & 0.5 & 0.9 & 1.8 & 1.8 \\
\hline Local planning & 5.2 & 2.8 & 6.0 & 2.8 & 6.2 & 4.4 & 3.2 & 2.2 & 5.2 & 2.4 \\
\hline Feedback processing & 0.6 & 1.0 & 5.4 & 4.8 & 17.8 & 12.1 & 30.5 & 23.4 & 13.6 & 9.0 \\
\hline Task-irrelevant cognitions & 1.5 & 1.5 & 1.8 & 2.7 & 0.7 & 1.2 & 0.6 & 0.8 & 1.1 & 1.0 \\
\hline Comments & 6.5 & 4.2 & 6.0 & 2.7 & 4.6 & 4.4 & 8.6 & 7.8 & 6.4 & 3.7 \\
\hline Solution development & 32.1 & 21.2 & 59.9 & 11.1 & 60.0 & 11.8 & 50.1 & 21.2 & 50.4 & 9.3 \\
\hline \multicolumn{11}{|l|}{ Moderate performers $(n=12)$} \\
\hline PC by requirements & 50.5 & 14.5 & 11.1 & 9.4 & 9.1 & 10.5 & 9.2 & 14.3 & 20.0 & 9.3 \\
\hline PC by scenarios & 6.4 & 3.8 & 8.1 & 10.8 & 5.4 & 5.9 & 2.1 & 2.4 & 5.5 & 3.6 \\
\hline Planning ahead & 1.8 & 2.1 & 2.2 & 2.8 & 1.1 & 1.5 & 1.6 & 2.3 & 1.7 & 1.5 \\
\hline Local planning & 5.0 & 2.0 & 3.0 & 1.9 & 4.3 & 3.2 & 3.5 & 2.6 & 3.8 & 1.8 \\
\hline Feedback processing & 0.4 & 1.0 & 1.3 & 2.2 & 8.2 & 11.2 & 15.8 & 16.4 & 6.5 & 6.7 \\
\hline Task-irrelevant cognitions & 2.6 & 3.8 & 7.6 & 2.7 & 4.9 & 5.9 & 7.3 & 10.7 & 5.6 & 6.3 \\
\hline Comments & 5.4 & 4.2 & 6.5 & 4.5 & 5.1 & 2.6 & 7.5 & 6.3 & 6.1 & 3.4 \\
\hline Solution development & 27.2 & 14.8 & 59.6 & 14.3 & 61.4 & 12.6 & 53.0 & 16.6 & 50.2 & 8.6 \\
\hline
\end{tabular}

Note. $\mathrm{PC}$ by requirements $=$ problem comprehension by analyzing requirements; PC by scenarios $=$ problem comprehension by scenarios. 
Table 3

Analysis of Variance for Verbal Protocol Categories

\begin{tabular}{lcccc}
\hline \multicolumn{1}{c}{ Measure } & $\begin{array}{c}\text { Performance level, } \\
F(1,22)\end{array}$ & $\begin{array}{c}\text { Phase, } \\
F(3,20)\end{array}$ & $\begin{array}{c}\text { Performance level } \\
\times \text { Phase, } F(3,20)\end{array}$ & $\begin{array}{c}\text { Effect size of } \\
\text { performance level }(d)\end{array}$ \\
\hline PC by requirements & $4.63^{*}$ & $64.62^{* *}$ & 1.23 & -.89 \\
PC by scenarios & 0.85 & $2.45^{\mathrm{a}}$ & 0.71 & .36 \\
Planning ahead & 0.06 & 1.65 & 1.36 & .07 \\
Local planning & 2.03 & $3.29^{*}$ & $3.09^{*}$ & .67 \\
Feedback processing & $4.99^{*}$ & $11.79^{* *}$ & $2.88^{\mathrm{b}}$ & .87 \\
Task-irrelevant cognitions & $5.80^{*}$ & 2.06 & 2.02 & -1.00 \\
Comments & 0.04 & 2.16 & 0.46 & .08 \\
Solution development & 0.01 & 0.01 & 0.17 & .02 \\
\hline
\end{tabular}

Note. $\mathrm{PC}$ by requirements $=$ problem comprehension by analyzing requirements; $\mathrm{PC}$ by scenarios $=$ problem comprehension by scenarios.

${ }^{\mathrm{a}} p=.093,{ }^{\mathrm{b}} p=.061$.

${ }^{*} p<.05 .{ }^{* *} p<.01$.

performers additionally differing within this course. Inspection of the means in local planning across the four phases suggests that high performers did more local planning in the second and third phase but not in the first and fourth phase. A comparison of the amount of local planning in the second and third phase between high and moderate performers supported this assumption, $t(22)=$ $2.18, p<.05, d=0.83$.

Hypothesis 3 , which predicted a higher amount of feedback processing in high performers compared with moderate performers, was supported. Additionally, the amount of feedback processing varied across the phases with generally more feedback processing in the later phases. The marginally significant interaction effect indicates that the difference between high and moderate performers increased over time.

As predicted in Hypothesis 4, analysis showed that high performers verbalized less task-irrelevant cognitions than did moderate performers.

Hypothesis 5 predicted that high performers would produce more visualizations than moderate performers. To test this hypothesis, problem visualizations and solution visualizations were examined separately. Analysis showed that nearly every participant produced problem visualizations ( $75 \%$ of high performers; $83 \%$ of moderate performers), $\chi^{2}(1, N=24)=0.25, n s, w=0.10$. For solution visualizations a significant difference was found with $58 \%$ of high performers sketching ideas and concepts concerning a possible solution compared to $8 \%$ of moderate performers, $\chi^{2}(1, N=24)=6.75, p<.01, w=0.53$. One might argue that moderate performers produced less solution visualizations because they had more difficulties with the task and did not know what to visualize. However, no significant difference was found in the total amount of notes taken by high performers $(M=8.3$ pages, $S D$ $=3.8)$ and moderate performers $(M=7.7$ pages, $S D=$ $2.8), t(22)=.43, n s, d=0.18$. This shows that the high performers' more extensive solution visualizations can not be explained by more note-taking activity in this group. Thus, Hypothesis 5 was partially supported.

Hypothesis 6 assumed more knowledge about strategies in high performers. Participants' knowledge was analyzed by asking them which higher order principles they would recommend to an imagined inexperienced colleague working on the lift control problem. High performers named 4.3 strategies ( $S D=1.2$ ) compared with 1.6 strategies ( $S D=1.4$ ) named by moderate performers, $t(22)$ $=5.02, p<.01, d=2.03$. One might argue that high performers did not know more but were superior in verbalizing their knowledge. However, analysis showed that high and moderate performers did not differ with respect to verbalization skills ( $M=3.7, S D=1.7$ for high performers; $M=3.1, S D=1.4$ for moderate performers), $t(22)=0.95, n s, d=0.40$. In an analysis of covariance (ANCOVA) with number of recommended strategies as dependent variable and verbalization skills as the covariate, the difference between high and moderate performers in recommended strategies remained significant, $F(1,21)$ $=22.56, p<.01$, providing clear support for Hypothesis 6 .

\section{Further Analyses}

Detailed analysis of problem comprehension. To analyze the problem comprehension process in more detail, I computed relative transition frequencies between "problem comprehension by analyzing requirements," "problem comprehension by scenarios," and other verbal protocol categories. These relative transition frequencies indicate how often each of the two problem comprehension categories were immediately followed by any other problem comprehension activity or any other category. On average, most requirement segments were immediately followed by another requirement segment $(M=73.8, S D$ 
$=11.9$ for high performers; $M=78.3, S D=7.1$ for moderate performers); most scenario segments were immediately followed by another scenario segment ( $M=$ $58.9, S D=22.4$ for high performers; $M=47.4, S D=$ 18.5 for moderate performers). There were no significant differences between high and moderate performers on either requirement, $t(22)=-1.12, n s$, or scenarios, $t(22)$ $=1.37, n s$. However, in the verbal protocols of high performers ( $M=4.4, S D=3.6$ ) a requirement segment was more frequently followed by a scenario segment than was the case for the protocols of moderate performers $(M=$ $2.0, S D=1.8), t(16.10)=2.04, p=.058, d=0.84$. This marginally significant difference remained stable in an ANCOVA with the amount of scenarios used as a covariate, $F(1,21)=3.10, p=.093$. This finding indicates that the problem comprehension process differed between high and moderate performers with high performers more often relating requirements immediately to lift domain scenarios.

Detailed analysis of task-irrelevant cognitions. To examine how task-irrelevant cognitions were embedded in the design process, I computed relative transition frequencies between task-irrelevant cognitions and other verbal protocol categories. Analysis showed that for high performers $25 \%$ ( $S D=24.3$ ) of all task-irrelevant cognitions were followed by another task-irrelevant cognition compared with $51 \%(S D=24.0)$ for moderate performers, $t(21)=-2.58, p<.01, d=-1.08)$. In high performers' verbal protocols $31 \%(S D=20.1)$ of all task-irrelevant cognitions were immediately followed by a statement indicating solution development compared to $16 \%(S D=$ 11.5 ) in moderate performers' protocols, $t(15.65)=2.14$, $p<.05, d=0.90$. There was no significant difference with respect to other verbal protocol categories $(M=$ 44.0, $S D=25.7$ for high performers; $M=32.7 ; S D=$ 25.0 for moderate performers), $t(21)=1.06, n s, d=$ 0.45 . This finding shows that moderate performers tended to dwell on their task-irrelevant cognitions, whereas high performers overcame these cognitions more quickly and continued to work on the design solution.

Content of recommended strategies. High and moderate performers were compared with respect to the following strategies named by at least $25 \%$ of the 40 participants: intensive problem comprehension, divide and conquer, and cooperation with colleagues. In the high-performance group, $67 \%$ of the participants recommended intensive problem comprehension as a useful strategy compared with $8 \%$ in the moderate performing group, $\chi^{2}(1, N=24)=$ $6.40, p<.05, w=0.60$. High performers also more often recommended cooperation with colleagues $(67 \%)$ than did moderate performers $(17 \%), \chi^{2}(1, N=24)=6.17, p<$ $.05, w=0.51$. No differences were found with respect to divide and conquer, recommended by $33 \%$ of high perform- ers and $25 \%$ of moderate performers, $\chi^{2}(1, N=24)=$ $0.00, n s, w=0.09$.

It might be suggested that the more frequent recommendation of cooperation with colleagues expressed by high performers is an artifact of the specific performance measure that was partly based on peer nominations. One might argue that software designers who regard cooperation as important, simply cooperated more extensively with their colleagues and therefore were nominated more often by their peers. To rule out this interpretation, I performed an additional analysis in which the total sample of 40 participants was split into two subgroups based on their design task performance. Participants with a design task performance above the median were assigned to the first subgroup. Participants with a design task performance below the median were assigned to the second subgroup. This assignment was done irrespective of the peer nomination outcome. Analysis showed that $47 \%$ of the participants with a design task performance above the median recommended cooperation with colleagues compared with $14 \%$ of the participants with a design task performance below the median, $\chi^{2}(1, N=40)=5.20, p<.05, w=$ .36. Thus, the more frequent recommendation of cooperation with colleagues expressed by high performers is not likely to be due to the peer-nomination method.

Length of experience. Length of experience did not differ between high ( $M=6.6, S D=1.8)$ and moderate performers $(M=7.8, S D=3.3), t(17.28)=-1.16, n s$, $d=-0.45$. ANCOVAs with length of experience as a covariate showed that none of the design process differences between high and moderate performers could be explained by length of experience. When length of experience was taken into account as a covariate, high performers again showed less problem comprehension by analyzing requirements, $F(1,21)=5.35, p<.05$, did more local planning during the second and third phase, $F(1$, $21)=4.50, p<.05$, showed more feedback processing, $F(1,21)=4.97, p<.05$, and verbalized fewer taskirrelevant cognitions, $F(1,21)=7.54, p<.05$. Similarly, an ANCOVA with number of recommended strategies as the dependent variable and length of experience as a covariate confirmed the significant differences between high and moderate performers, $F(1,21)=22.67, p<.01$, found in the ANOVA. No significant effect of the covariate length of experience on any verbal protocol category or number of recommended strategies was found.

\section{Discussion}

The study showed that high and moderate performers differed with respect to action processes and knowledge. High performers spent more time on feedback processing (Hypothesis 3) and less time on task-irrelevant cognitions (Hypothesis 4). They produced more solution visualiza- 
tions (Hypothesis 5 ) and knew more about strategies ( $\mathrm{Hy}$ pothesis 6 ). However, there was only partial support for the hypothesis that high performers spend more time on planning (Hypothesis 2). High performers did not spend more time on problem comprehension early in the process (Hypothesis 1).

Across all phases, high performers spent less time analyzing requirements presented in the task description than did moderate performers. This finding suggests that high performers were able to build an adequate problem representation very early in the design process, which shortened subsequent problem comprehension activities. Similarly, previous research identified adequate problem representation as typical for high performers (de Groot, 1978; Green \& Gilhooly, 1992; Klein \& Hoffman, 1993). From the present study one can conclude that relating requirements to the problem domain scenarios plays an important role in arriving quickly at an adequate problem representation.

However, other studies reported more problem comprehension activities of high performers (Klemp \& McClelland, 1986; Lesgold et al., 1988; Vessey, 1986). One reason for the inconsistency between present and earlier research can be seen in the task description used. Although the lift control problem was a complex task, relatively little information was provided in the task description itself. Therefore, reading the task description more often did not offer additional information.

High performers verbalized more local planning in the second and third phase than did moderate performers. While working on the task, high performers briefly stated what they intended to do next, for example: "This point I have to describe in more detail, there is more behind it" or "'Okay, now I first do my priority module." Thus, without extensive elaboration, high performers made their intentions concerning the next step explicit. It seems that local planning served as an economic way of structuring the course of action, particularly in the two middle phases of the design process that were mainly dominated by solution development.

High performers did not show more planning ahead than moderate performers. Furthermore, the percentage of planning ahead was very small for both performance groups. This is in accordance with a study by Voss et al. (1983) that reported that experienced political scientists did not articulate higher level plans when working on a problem within their domain of expertise. An explanation for these findings is that professionals refer to heuristics and ready-made action plans and, therefore, do not need to plan explicitly in advance (Sonnentag, 1996). Presumably both high and moderate performers rely on such readymade action plans because both have acquired routines during their professional experience. However, earlier studies found more planning activities in high performers than in moderate or low performers (e.g., Dörner et al., 1983; Klemp \& McClelland, 1986). These studies used complex simulations and managerial tasks that require a high amount of planning ahead. In contrast, design tasks as used in the present study require less planning because they do not need to be accomplished in a fixed sequence (cf. Guindon, 1990).

High performers spent twice as much time on feedback processing than did moderate performers. As was reported with respect to visualizations, high performers did not have more material available for feedback processing. They made more use of it. Feedback processing might not only have a short-term effect immediately reflected in design task performance. The availability of task-related feedback also has a positive effect on learning and on future task performance (Ericsson \& Lehmann, 1996; Kluger \& DeNisi, 1996). This suggests that high performers who actively evaluate their design solutions not only perform better at the present task but also gain from it for the future. In the long run, feedback processing might result in high performers getting further ahead of moderate performers.

High performers verbalized fewer task-irrelevant cognitions than did moderate performers. Furthermore, when verbalizing such thoughts high performers returned more often immediately to solution development compared with moderate performers who continued to dwell on taskirrelevant cognitions. It might be that high performers pursued more specific goals (Hershey et al., 1990) that helped in avoiding and overcoming task-irrelevant cognitions (Lord \& Levy, 1994).

The study provides a detailed answer to the controversy about whether high or moderate performers use more visualizations in their working process (Adelson \& Soloway, 1988; Chi et al., 1982; Davies, 1992). Problem visualizations were widely used among all participants - irrespective of their performance level. When relating this finding to the verbal protocol data, one might conclude that only high performers gained from problem visualizations, whereas moderate performers did not. High performers produced solution visualizations more often than did moderate performers. This finding suggests that these visualizations are a helpful cognitive tool in the solution development process.

The study showed that high performers knew more about strategies than did moderate performers. This difference could not be explained by differences in verbalization skills. High performers particularly stressed the importance of intensive problem comprehension and cooperation with colleagues. When integrating these findings with the results of verbal protocol analysis, one can conclude that recommending intensive problem comprehension does not imply a need to spend much time on problem comprehension, but rather to apply a successful problem 
comprehension strategy. Previous expertise research focusing mainly on cognitive processes has not paid much attention to cooperation in work settings. However, some field studies demonstrated the importance of cooperation for high performance within software design and related fields (Curtis, Krasner, \& Iscoe, 1988; Kelley \& Caplan, 1994; Sonnentag, 1995). By following a different methodological procedure, the present study supports these field study findings and suggests that cooperation plays a greater role for high performance than shown in previous expertise studies.

Length of experience did not explain performance differences within this sample of professionals. Thus, the study suggests that within expertise research it makes a difference if one compares students with professionals or if one studies differences within samples of professionals. Comparisons between students and professionals indicated that differences in length of experience can explain performance differences (Adelson, 1981; Eteläpelto, 1993; Jeffries et al., 1981). However, this is not the case for professionals. This implies that results of many expertise studies based on comparisons between students and professionals may not be generalized to expertise among professionals.

Nevertheless, this interpretation should not lead to the conclusion that experience is irrelevant for expertise. Studies suggest that other aspects of experience, such as its variety (Sonnentag, 1995; Voss et al., 1983), cumulative amount of deliberate practice (Ericsson et al., 1993), or mastered challenges (Klein \& Hoffman, 1993), are more important than length of experience. Furthermore, the present finding is compatible with a threshold model (cf. Schneider, 1993, for a related idea) that claims that experience matters up to a certain level but once that level is reached, other factors become more important.

Differences between high and moderate performers were not restricted to one isolated element of the action process. High performers mastered the action process as a whole, ranging from problem comprehension to feedback processing. In summary, they demonstrated a solutionorientated working style throughout the whole process. Manifestations of this working style were refraining from task-irrelevant cognitions, quickly returning to solution developments, using visualizations to support solution development, local planning during solution development, and feedback processing.

\section{Strengths and Limitations}

Expertise was measured with both an internally and externally valid performance measure. Combining these two performance measures ensured that participants referred to as high performers really outperformed moderate performers in a standardized situation (Ericsson \& Smith, 1991a) and that these performance differences were not restricted to one specific task in a laboratory setting, but that they existed as well within participants' everyday work situations.

One might question whether all study participants identified as high performers were really experts. For example, Ford and Kraiger (1995) pointed out that high performance and a well-organized domain-specific knowledge base are necessary constituents of the expertise concept. Within the present study, the possession of such a superior knowledge base was no precondition for being identified as a high performer (i.e., expert). However, hypothesis testing showed that high performers did have more knowledge about strategies. This finding suggests that the present study's high performers came close to more specific expertise criteria such as recommended by Ford and Kraiger. In further studies, one might consider using both the high performance and knowledge criterion for identifying experts. However, if this were done, it would preclude the use of knowledge as a dependent variable.

Until now only a limited number of studies analyzed problem comprehension, planning, feedback processing, and task-irrelevant cognitions over the course of the action process (e.g., Hershey et al., 1990; Simmons \& Lunetta, 1993). Most studies focused on measures aggregated across longer time intervals (e.g., Isenberg, 1986; Vitalari \& Dickson, 1983). By taking an explicit process perspective and analyzing verbal protocol data at a finegrained level, the present study demonstrated that high and moderate performers do not only differ with respect to the total amount of specific activities but also with respect to process features. Protocol analysis literature has stressed that analysis should be limited to task-relevant verbalizations (Ericsson \& Simon, 1993). This approach might be appropriate for a range of purposes. However, the present study demonstrated that some of the crucial differences between high and moderate performers only became evident when extending the analysis to task-irrelevant verbalizations.

An obvious limitation of the study is its sample size. In most of the analyses, 12 high performers were compared with 12 moderate performers. Without doubt, it is necessary to replicate the findings in a larger sample. However, it should be kept in mind that significant differences were found between high and moderate performers despite the small sample size. This corresponds to the large effect sizes found in the study. Other, highly cited studies offering important insights into the expertise phenomenon were also based on small (or even smaller) sample sizes. For example, de Groot (1978) studied 22 chess players and based his analyses on a total of 10 persons; Jeffries et al.'s (1981) sample was composed of 4 experienced software designers and 6 less experienced participants; Chi et al. ( 1982) compared no more than 8 experts 
with 8 nonexperts. Thus, for economy reasons, a small sample size is an inevitable drawback of studying real world experts. However, despite such relatively small sample sizes, crucial differences between high and moderate performers were uncovered.

\section{Consequences for Practice and Future Research}

This study has practical implications for training and personnel selection. Trainees should learn a solution-oriented working style incorporating local planning, visualizing design ideas during solution development, processing feedback by evaluating design solutions, and refraining from task-irrelevant cognitions. In addition, meta-cognitive skills should be taught ( Kraiger, Ford, \& Salas, 1993). Working style and cognitive processes during task accomplishment can be also used as predictors in personnel selection. Design tasks and other complex problem-solving tasks might be appropriate for assessing working style and cognitive processes (Funke, 1995; Putz-Osterloh, 1993). Length of professional experience was not related to effective design processes or to professional performance. Therefore, given that all applicants already have some professional experience, personnel selection decisions should not be based on length of experience.

The differences found between high and moderate performers clearly indicate that it makes sense to extend expertise research to professional settings. Methods such as verbal protocol analysis and knowledge elicitation stemming from cognitive psychology offer important insights into what distinguishes high and moderately performing professionals. To uncover the breadth and richness of high performers' action processes, I believe that it is important to have participants work on a complex task. Only then can it become obvious how high performers approach typical tasks and how they unfold their competencies during task accomplishment. When working on simple tasks, both high and moderate performers might have standard procedures available that do not differentiate between the two performance groups.

A crucial question to be addressed in future research refers to the predictors of superior action processes and knowledge. Cognitive abilities and achievement motivation might belong to such predictors (Gottfredson, 1986; Humphreys \& Revelle, 1984). Future research has to answer if and how various aspects of experience other than length contribute to outstanding performance.

The finding that high and moderate performers differed in action processes and knowledge might not come as a surprise to scientists and practitioners within industrial and organizational psychology. However, the results question the long tradition in more fundamental expertise research in which all professionals were regarded to be experts. It became obvious that the expertise phenomenon can not be explained by simply referring to length of experience but calls for a deeper look. The present study is a first step in this direction.

\section{References}

Adelson, B. (1981). Problem solving and the development of abstract categories in programming languages. Memory \& Cognition, 9, 422-433.

Adelson, B., \& Soloway, E. (1988). A model of software design. In M. T. H. Chi, R. Glaser, \& M. J. Farr (Eds.), The nature of expertise (pp. 185-208). Hillsdale, NJ: Erlbaum.

Anzai, Y. (1991). Learning and use of representations for physics expertise. In K. A. Ericsson \& J. Smith (Eds.), Toward a general theory of expertise: Prospects and limits (pp. 6492 ). Cambridge, England: Cambridge University Press.

Bellamy, R. K. E. (1994). What does pseudo-code do? A psychological analysis of the use of pseudo-code by experienced programmers. Human-Computer Interaction, 9, 225-246.

Black, A. (1990). Visible planning on paper and on screen: The impact of working medium on decision-making by novice graphic designers. Behaviour \& Information Technology, 9 , $283-296$.

Campbell, J. P., McCloy, R. A., Oppler, S. H., \& Sager, C. E. (1993). A theory of performance. In E. Schmitt, W. C. Borman, \& Associates (Eds.), Personnel selection in organizations (pp. 35-70). San Francisco: Jossey-Bass.

Carver, C. S., \& Scheier, M. F. (1982). Control theory: A useful conceptual framework for personality-social, clinical, and health psychology. Psychological Bulletin, 92, 111-135.

Charness, N., Krampe, R., \& Mayr, U. (1996). The role of practice and coaching in entrepreneurial skill domains: An international comparison of life-span chess skill acquisition. In K. A. Ericsson (Ed.), The road to excellence: The acquisition of expert performance in the arts and sciences, sports and games (pp. 51-80). Mahwah, NJ: Erlbaum.

Chase, W. G., \& Simon, H. A. (1973). The mind's eye in chess. In W. G. Chase (Ed.), Visual information processing (pp. 215-281). New York: Academic Press.

Chi, M. T. H., Glaser, R., \& Farr, M. J. (Eds.). (1988). The nature of expertise. Hillsdale, NJ: Erlbaum.

Chi, M. T. H., Glaser, R., \& Rees, E. (1982). Expertise in problem solving. In R. J. Sternberg (Ed.), Advances in the psychology of human intelligence (pp. 7-75). Hillsdale, NJ: Erlbaum.

Curtis, B., Krasner, H., \& Iscoe, N. (1988). A field study of the software design process for large systems. Communications of the ACM, 31, 1268-1287.

Davies, S. P. (1992). The role of expertise in the development of display-based problem solving strategies. Proceedings of the 14th Annual Conference of the Cognitive Science Society, 797-802. Hillsdale, NJ: Erlbaum.

de Groot, A. D. (1978). Thought and choice in chess (2nd ed.). The Hague, the Netherlands: Mouton.

Dörner, D., Kreuzig, H. W., Reither, F., \& Stäudel (Eds.). (1983). Lohhausen. Vom Umgang mit Unbestimmtheit und Komplexität [Lohhausen. On dealing with uncertainty and complexity ]. Bern, Switzerland: Huber.

Dörner, D., \& Schölkopf, J. (1991). Controlling complex sys- 
tems; or expertise as "grandmother's know-how." In K. A. Ericsson \& J. Smith (Eds.), Toward a general theory of expertise: Prospects and limits (pp. 218-239). Cambridge, England: Cambridge University Press.

Earley, P. C., Wojnaroski, P., \& Prest, W. (1987). Task planning and energy expended: Exploration of how goals influence performance. Journal of Applied Psychology, 72, 107-114.

Ericsson, K. A., Krampe, R. T., \& Tesch-Römer, C. (1993). The role of deliberate practice in the acquisition of expert performance. Psychological Review, 100, 363-406.

Ericsson, K. A., \& Lehmann, A. C. (1996). Expert and exceptional performance: Evidence of maximal adaptation to task constraints. Annual Review of Psychology, 47, 273-305.

Ericsson, K. A., \& Simon, H. A. (1993). Protocol analysis. Verbal reports as data (rev. ed.). Cambridge, MA: Massachusetts Institute of Technology.

Ericsson, K. A., \& Smith, J. (1991a). Prospects and limits of the empirical study of expertise: An introduction. In $\mathrm{K}$. A. Ericsson \& J. Smith (Eds.), Toward a general theory of expertise: Prospects and limits (pp. 1-38). Cambridge, England: Cambridge University Press.

Ericsson, K. A., \& Smith, J. (Eds.). (1991b). Toward a general theory of expertise. Cambridge, England: Cambridge University Press.

Eteläpelto, A. (1993). Metacognition and the expertise of computer program comprehension. Scandinavian Journal of Educational Research, 37, 243-254.

Ford, J. K., \& Kraiger, K. (1995). The application of cognitive constructs and principles to the instructional systems model of training: Implications for needs assessment, design, and transfer. In C. L. Cooper \& I. T. Robertson (Eds.), International Review of Industrial and Organizational Psychology (pp. 1-48). Chichester, England: Wiley.

Frese, M., \& Zapf, D. (1994). Action as the core of work psychology: A German approach. In H. C. Triandis, M. D. Dunnette, \& L. M. Hough (Eds.), Handbook of industrial and organizational psychology (Vol. 4, 2nd ed., pp. 271340). Palo Alto, CA: Consulting Psychologists Press.

Funke, U. (1995). Using complex problem solving tasks in personnel selection and training. In P. A. Frensch \& J. Funke (Eds.), Complex problem solving. The European perspective (pp. 219-240). Hillsdale, NJ: Erlbaum.

Glaser, R., \& Chi, M. T. H. (1988). Overview. In M. T. H. Chi, R. Glaser, \& M. J. Farr (Eds.), The nature of expertise (pp. XV-xxviii). Hillsdale, NJ: Erlbaum.

Gollwitzer, P. M. (1993). Goal achievement: The role of intentions. In W. Stroebe \& M. Hewstone (Eds.), European review of social psychology (Vol. 4, pp. 141-185). London: Wiley.

Gottfredson, L. S. (1986). Societal consequences of the $g$ factor. Journal of Vocational Behavior, 29, 379-410.

Green, A. J. K., \& Gilhooly, K. J. (1992). Empirical advances in expertise research. In M. T. Keane \& K. J. Gilhooly (Eds.), Advances in the psychology of thinking (pp. 45-70). New York: Harvester Wheatsheaf.

Guindon, R. (1990). Designing the design process: Exploiting opportunistic thoughts. Human-Computer Interaction, 5, 305-344.

Hacker, W. (1986). Arbeitspsychologie [Work psychology]. Bem, Switzerland: Huber.
Hacker, W. (1992). Expertenkönnen: Erkennen und Vermitteln [Expert competence: Assessment and instruction]. Göttingen, Germany: Verlag für Angewandte Psychologie.

Hayes-Roth, B., \& Hayes-Roth, F. (1979). A cognitive model of planning. Cognitive Science, 3, 275-310.

Hershey, D. A., Walsh, D. A., Read, S. J., \& Chulef, A. S. (1990). The effects of expertise on financial problem solving: Evidence for goal-directed, problem-solving scripts. Organizational Behavior and Human Decision Processes, 46, $77-$ 101.

Humphreys, M. S., \& Revelle, W. (1984). Personality, motivation, and performance: A theory of the relationship between individual differences and information processing. Psycholog ical Review, 91, 153-184.

Isenberg, D. J. (1986). Thinking and managing: A verbal protocol analysis of managerial problem solving. Academy of Management Journal, 29, 775-788.

Jeffries, R., Turner, A. A., Polson, P. G., \& Atwood, M. E. (1981). The processes involved in designing software. In J. R. Anderson (Ed.), Cognitive skills and their acquisition (pp. 255-283). Hillsdale, NJ: Erlbaum.

Kelley, R., \& Caplan, J. (1994). Wie die Bell Laboratories die Leistung ihrer Stars verbessern [How Bell Laboratories improve their stars' performance]. Harvard Business Manager, 2, 33-42.

Klein, G. A., \& Hoffman, R. R. (1993). Seeing the invisible: Perceptual-cognitive aspects of expertise. In M. Rabinowitz (Ed.), Cognitive science: Foundations of instruction (pp. 203-226). Hillsdale, NJ: Erlbaum.

Klemp, G. O., \& McClelland, D. C. (1986). What characterizes intelligent functioning among senior managers? In R. J. Sternberg \& R. K. Wagner (Eds.), Practical intelligence: Nature and origin of competence in the everyday world (pp. $31-$ 50). Cambridge, England: Cambridge University Press.

Kluger, A. N., \& DeNisi, A. (1996). The effects of feedback interventions on performance: A historical review, a metaanalysis, and a preliminary feedback intervention theory. $P$ sychological Bulletin, 119, 254-284.

Koubek, R. J., \& Salvendy, G. (1991). Cognitive performance of super-experts on computer program modification tasks. Ergonomics, 34, 1095-1112.

Kraiger, K., Ford, J. K., \& Salas, E. (1993). Application of cognitive, skill-based, and affective theories of learning outcomes to new methods of training evaluation. Journal of Applied Psychology, 78, 311-328.

Landis, J. R., \& Koch, G. G. (1977). The measurement of observer agreement for categorical data. Biometrics, 33, 159174.

Larkin, J. H., \& Simon, H. A. (1987). Why a diagram is ( sometimes) worth ten thousand words. Cognitive Science, 11, 6599.

Lesgold, A., Rubinson, H., Feltovich, P., Glaser, R., Klopfer, D., \& Wang, Y. (1988). Expertise in a complex skill: Diagnosing X-ray pictures. In M. T. H. Chi, R. Glaser, \& M. J. Farr (Eds.), The nature of expertise (pp. 311-342). Hillsdale, NJ: Erlbaum.

Lord, R. G., \& Levy, P. E. (1994). Moving from cognition to action: A control theory perspective. Applied Psychology: An International Review, 43, 335-398. 
McKeithen, K. B., Reitman, J. S., Rueter, H. H., \& Hirtle, S. C. (1981). Knowledge organization and skill differences in computer programmers. Cognitive Psychology, 13, 307-325.

Mikulincer, M. (1994). Human learned helplessness: A coping perspective. New York: Plenum.

Mikulincer, M., Kedem, P., \& Zilcha-Segal, H. (1989). Learned helplessness, reactance and cue utilization. Journal of Research in Personality, 23, 235-247.

Miller, G. A., Galanter, E., \& Pribram, K. (1960). Plans and the structure of behavior. New York: Holt, Rinehart \& Winston.

Patel, V. L., \& Groen, G. J. (1991). The general and specific nature of medical expertise: A critical look. In K. A. Ericsson \& J. Smith (Eds.), Toward a general theory of expertise (pp. 93125 ). Cambridge, England: Cambridge University Press.

Putz-Osterloh, W. (1993). Complex problem solving as a diagnostic tool. In H. Schuler, J. L. Farr, \& M. Smith (Eds.), Personnel selection and assessment. Individual and organizational perspectives (pp. 289-301). Hillsdale, NJ: Erlbaum.

Reif, F., \& Allen, S. (1990). Cognition for interpreting scientific concepts: A study of acceleration. Cognition and Instruction, 9, $1-44$.

Rohwer, W. D., \& Thomas, J. W. (1989). The role of autonomous problem-solving activities in learning to program. Journal of Educational Psychology, 81, 584-593.

Schaub, H., \& Strohschneider, S. (1992). Die Auswirkungen unterschiedlicher Problemlöseerfahrung mit einem unbekannten komplexen Problem [ Effects of different experiences with problems on how to deal with an unknown complex problem]. Zeitschrift für Arbeits- und Organisationspsychologie, $36,117-126$.

Schneider, W. (1993). Acquiring expertise: Determinants of exceptional performance. In K. A. Heller, F. J. Mönks, \& A. H. Passow (Eds.), International handbook of research and development of giftedness and talent (pp. 311-324). Oxford, England: Pergamon Press.
Shanteau, J. (1988). Psychological characteristics and strategies of expert decision makers. Acta Psychologica, 68, 203-215.

Simmons, P. E., \& Lunetta, V. N. (1993). Problem-solving behaviors during a genetics computer simulation: Beyond the expert/novice dichotomy. Journal of Research in Science Teaching, 30, 153-173.

Soloway, E., Adelson, B., \& Ehrlich, K. (1988). Knowledge and processes in the comprehension of computer programs. In M. T. H. Chi, R. Glaser, \& M. J. Farr (Eds.), The nature of expertise (pp. 129-152). Hillsdale, NJ: Erlbaum.

Sonnentag, S. (1995). Excellent software professionals: Experience, work activities, and perceptions by peers. Behaviour \& Information Technology, 14, 289-299.

Sonnentag, S. (1996). Planning and knowledge about strategies: Their relationship to work characteristics in software design. Behavior \& Information Technology, 15, 213-225.

Vessey, I. (1986). Expertise in debugging computer programs: An analysis of the content of verbal protocols. IEEE Transactions on Systems, Man, and Cybernetics, 16, 621-637.

Vitalari, N. P., \& Dickson, G. W. (1983). Problem solving for effective systems analysis: An experimental exploration. Communications of the ACM, 26, 948-956.

Voss, J. F., Greene, T. R., Post, T. A., \& Penner, B. C. (1983). Problem-solving skill in the social sciences. In G. H. Bower (Ed.), The psychology of learning and motivation. Advances in research and theory (pp. 165-213). New York: Academic Press.

Wiggins, M., \& O'Hare, D. (1995). Expertise in aeronautical weather-related decision making: A cross-sectional analysis of general aviation pilots. Journal of Experimental Psychology: Applied, $1,305-320$.

Wolff, S. (1989). Knowledge acquisition and possibilities for elicitating expert knowledge. In F. Klix, N. A. Streitz, Y. Waern, \& H. Wandtke (Eds.), Man-computer interaction research MACINTER II (pp. 413-421). Amsterdam: Elsevier.

\section{Appendix}

\section{The Lift Control Problem (Guindon, 1990)}

A system with $\mathrm{N}$ lifts has to be installed in a building with $\mathrm{M}$ floors. The lifts and the control mechanism are supplied by a manufacturer. The internal mechanisms of the lift system are described below. Design the logic to move lifts between floors in the building according to the following rules:

1. Each lift has a set of buttons, one button for each floor. These buttons illuminate when pressed and cause the lift to visit the corresponding floor. The illumination is cancelled when the corresponding floor is stopped at by the lift.

2. Each floor has two buttons (except ground and top), one to request an up lift and one to request a down lift. These buttons illuminate when pressed. The buttons are cancelled when a lift visits the floor and is either travelling in the desired direction or visiting the floor with no request outstanding. In the latter case, if both floor request buttons are illuminated, only one should be cancelled. The algorithm used to decide which request to service first should minimize the waiting time for both requests.

3. When a lift has no requests to service, it should remain at its final destination with its doors closed and await further requests - or model a 'holding' floor.

4. All requests for lifts from floors must be serviced eventually, with all floors given equal priority. Can this be proved or demonstrated?

5. All requests for floors within lifts must be serviced eventually, with floors being service sequentially in the direction of travel. Can this be proved or demonstrated?

6. Each lift has an emergency button, that, when pressed, causes a warning signal to be sent to the site manager. The lift is then deemed "out of service." Each lift has a mechanism to cancel its out-of-service status.

From "Designing the design process: Exploiting opportunistic thoughts," by R. Guindon, 1990, Human-Computer Interaction, 5, p. 314. Copyright 1990 by Erlbaum. Reprinted with permission. 